\title{
Renal Effects of Antisense-Mediated Inhibition of SGLT2 [
}

\author{
Leonie van Meer, Matthijs Moerland, Marloes van Dongen, Bas Goulouze, Marieke de Kam, \\ Erica Klaassen, Adam Cohen, and Jacobus Burggraaf
}

Centre for Human Drug Research, Leiden, The Netherlands

Received March 23, 2016; accepted August 22, 2016

\section{ABSTRACT}

ISIS 388626 is an antisense sodium-glucose cotransporter 2 (SGLT2) inhibitor designed to treat type 2 diabetes mellitus by induction of glucosuria. ISIS 388626 was demonstrated to be safe and effective in preclinical trails in several species. We undertook the present study to evaluate the safety and efficacy of 13 weekly doses of 50,100 , and $200 \mathrm{mg}$ of ISIS 388626 in humans. ISIS 388626 increased 24-hour urinary glucose excretion dose dependently with $508.9 \pm 781.45 \mathrm{mg} /$ day in the $100-\mathrm{mg}$ and $1299.8 \pm 1833.4 \mathrm{mg} /$ day in the $200-\mathrm{mg}$ cohort, versus $88.7 \pm 259.29 \mathrm{mg} /$ day in the placebo group. ISIS 388626 induced a reversible increase in serum creatinine, with the largest effect after eight doses of ISIS $388626(200 \mathrm{mg}$; $0.38 \pm 0.089 \mathrm{mg} / \mathrm{dl} ; 44 \%$ increase over baseline). Three subjects were discontinued as a result of creatinine increases. The renal clearance test revealed no indications for impairment of glomerular filtration or renal perfusion. The creatinine increases were accompanied by a rise in the levels of urinary renal damage markers $[\beta-2-$ microglobulin (B2M), total protein, kidney injury molecule (KIM1), $\alpha$-glutathione $S$-transferase (aGST), $N$-acetyl- $\beta$-(D)-glucosaminidase (NAG)]. Other treatmentrelated adverse events included mild injection site reactions occurring in $8-19 \%$ of the subjects. In conclusion, ISIS 388626 treatment induced glucosuria at a dose level of $200 \mathrm{mg} /$ week. This intended pharmacological effect was small, amounting to approximately $1 \%$ of the total amount of filtered glucose. Changes in serum and urinary markers were indicative of transient renal dysfunction, most probably of tubular origin. Whether the glucosuria is caused by specific SGLT2 inhibition or general tubular dysfunction or a combination remains uncertain.

\section{Introduction}

The antisense oligonucleotide ISIS 388626 inhibits the synthesis of the renal antisense sodium-glucose cotransporter 2 (SGLT2) receptor, which accounts for $90 \%$ of the reabsorption of glomerular filtrated glucose (Zanardi et al., 2012; Bhanot, 2009). SGLT2 inhibition affects renal glucose reabsorption, resulting in glucosuria and lowered serum glucose levels. The strategy of SGLT2 inhibition for the treatment of type 2 diabetes mellitus is efficacious and has led to the registration and approval of a number of small-molecule SGLT2 inhibitors (Plosker, 2012; Jahagirdar and Barnett, 2014; Sha et al., 2014). An alternative to SGLT2 inhibition by small molecules, which results in a moderate $30-50 \%$ inhibition only, could be antisense-mediated SGLT2 knockdown. ISIS 388626 is such an antisense oligonucleotide; in animal models it causes $\geq 80 \%$ reduction in renal SGLT2 mRNA expression at doses of $1-3 \mathrm{mg} / \mathrm{kg}$ per week (rodents) to $30 \mathrm{mg} / \mathrm{kg}$ per week (monkeys), resulting in effective glucosuria (Bhanot, 2009; Zanardi et al., 2012).

As ISIS 388626 appeared to be effective and safe in animal studies ranging from 6 weeks to 6 months in duration the compound was tested in humans. These first clinical studies-

dx.doi.org/10.1124/jpet.116.233809.

S This article has supplemental material available at jpet.aspetjournals.org. with 50-100 mg s.c. weekly after a loading-dose regimen of three doses during the first week-showed, contrary to expectations, possibe untoward renal effects (unpublished, L. van Meer et al.). In summary, 3-4 weeks of treatment with ISIS 388626 resulted in dose-dependent, transient, fully reversible, and variable (range: $0-73 \%$ at $100 \mathrm{mg}$ of ISIS 388626) increases in serum creatinine accompanied by increased urinary excretion of renal markers such as $\beta-2$ microglobulin (B2M) and kidney injury molecule 1 (KIM1). This was unexpected, as animals treated with $0,1,3,10$, or $30 \mathrm{mg} / \mathrm{kg}$ per week, with assessments at 6 and 13 weeks, had not shown such results. The pertinent clinical study was halted early and further preclinical data were collected. First, bio-banked samples collected earlier than 6 weeks in previous preclinical experiments were analyzed. This showed also that relatively early during weekly treatment with ISIS 388626 after using loading doses in animals, there were transient increases in serum creatinine and urinary excretion of B2M and protein. The changes were reversible even upon continuation of dosing (unpublished data, on file). Thus, the apparent discrepancy in renal effects of ISIS 388626 treatment in rodent, monkey, and human could be explained by the timing of the assessments (Wancewicz, 2008; Bhanot, 2009; Zanardi et al., 2012 ). A further dedicated experiment in monkeys explored whether the renal effects by ISIS 388626 could be explained by the loading dose. Animals were dosed for

ABBREVIATIONS: 2' MOE, 2'O-methyl; AEs, adverse events; aGST, $\alpha$-glutathione $S$-transferase; AUC, area under the curve; B2M, $\beta$-2microglobulin; GFR, glomerular filtration rate; ISR, injection site reaction; KIM1, kidney injury molecule; NAG, $N$-acetyl- $\beta$-(D)-glucosaminidase; OGTT, oral glucose tolerance test; PAH, para-aminohippurate; RPF, renal plasma flow; SGLT2, antisense sodium-glucose cotransporter 2. 
13 weeks with either $30 \mathrm{mg} / \mathrm{kg}$ every other day or a single dose in the first week, followed by weekly dosing for another 12 weeks. This experiment showed that changes in renal markers occurred only with the loading dose regimen. Importantly, this study also showed that abandoning the loading dose ISIS 388626 still resulted in glucosuria, whereas changes in serum creatinine did not occur.

On the basis of these findings, the clinical study was restarted with the aim to investigate the effects of 13 weekly s.c. doses of 50, 100, and $200 \mathrm{mg}$ of ISIS 388626 . The study design included oral glucose tolerance tests (OGTT) to estimate the intended pharmacodynamic effect (the induction of glucosuria) and close monitoring of renal function and injury, with application of strict predefined stopping criteria. However, as described here, omitting the loading dose did not prevent increases in renal markers at a dose level of $50 \mathrm{mg}$. Therefore further dose escalation was postponed and the 50-mg cohort was repeated in order to investigate whether the transient increases in renal markers in humans could be explained by functional changes in renal blood flow and/or glomerular filtration rate. This paper reports on the findings from these clinical experiments and the continuation with the 100- and 200-mg dose levels.

\section{Materials and Methods}

Subjects. Adult subjects (18-65 years), male or female (postmenopausal or surgically sterile), with BMI $<30 \mathrm{~kg} / \mathrm{m}^{2}$ and a fasting plasma glucose and $\mathrm{HbA} 1 \mathrm{C}$ below the upper limit of normal could participate in this study. Significant abnormalities in medical history, physical examination, 12-lead electrocardiogram, and clinical laboratory evaluations [including positive protein in urine dipstick analysis and calculated estimated glomerular filtration rate below $60 \mathrm{ml} / \mathrm{min}$ by the modification of diet in renal disease (MDRD) equation (Levey et al., 1999)] led to exclusion. The study was conducted in accordance with good clinical practice guidelines, after approval by the national ethics committee.

Study Design. This was a double-blind, randomized, placebocontrolled multiple-ascending dose study of 12 weeks in duration with 5 weeks of follow-up, using weekly administration of ISIS 388626 to establish the safety profile and pharmacodynamics of the compound, and performed at the Centre for Human Drug Research in the Netherlands. Per cohort, 16 randomly assigned subjects received multiple doses of either ISIS 388626 or placebo (in a 3:1 ratio), administered as subcutaneous injection. An OGTT was performed before the first administration of ISIS 388626 (or matching placebo) and after the 9 th and 13 th doses. The OGTT consisted of ingestion of a $75-\mathrm{mg}$ glucose solution, given after an overnight fast. Subsequently, blood was drawn regularly during 4 hours for determination of glucose, insulin, and C-peptide concentrations. The plan was to investigate the effects of 50,100 , and $200 \mathrm{mg}$ of ISIS 388626, but owing to unexpected findings in the 50-mg cohort, execution of the 100and 200-mg cohorts was postponed. An additional cohort was treated with 13 weekly doses of $50 \mathrm{mg}$ of ISIS 388626 or placebo to explore the nature of the observed safety signals in more detail.

Sample Size. The selection of a total of 12 subjects per treatment group was made on the basis of previous data obtained after conducting an OGTT in normal subjects. It is estimated that the standard deviation of change in plasma glucose area under the curve (AUC) for 0-120 minutes during the OGTT is approximately $85 \mathrm{mmol} \times \mathrm{min} / \mathrm{l}$. With six subjects in the pooled placebo group and six subjects in the ISIS 388626-treated group, this would result in at least $80 \%$ power to detect a $170-\mathrm{mmol} \times \mathrm{min} / \mathrm{l}$ difference in plasma glucose $\mathrm{AUC}_{0-120}$ minutes at an alpha level of 0.05 . Additional subjects were also included to ensure sufficient power of the efficacy analysis in case there was more-than-expected variation.
Dose Rationale. The plan was to explore 50, 100, and $200 \mathrm{mg}$ of ISIS 388626. Doses were calculated on the basis of a minimum anticipated biological effect approach, taking into account a no-adverseeffect level estimated to be $10 \mathrm{mg} / \mathrm{kg}$ per week (including a loading dose regimen) in monkeys. In preclinical studies across multiple species, the pharmacologically active dose range of ISIS 388626 was $1-3 \mathrm{mg} / \mathrm{kg}$ per week. At this exposure, a significant reduction in SGLT2 mRNA occurred (74 to $97 \%$ in mice and approximately 30 to $90 \%$ in monkeys over the dose range $1-30 \mathrm{mg} / \mathrm{kg}$ per week), accompanied by a 25 - to 200 -fold increase in urinary glucose excretion (Wancewicz, 2008; Bhanot, 2009; Zanardi et al., 2012). On this basis, estimation of the equivalent human effective dose fell in the range of 1-3 mg/kg per week. Experience with other 2'-MOE-modified antisense oligonucleotides, safely administered (intravenously and subcutaneously) in multiple clinical studies at doses up to weekly $750 \mathrm{mg}$ (which translates into $10.7 \mathrm{mg} / \mathrm{kg}$ per week assuming an average weight of $70 \mathrm{~kg}$ ), with treatment durations exceeding 1 year (Kwoh and Crooke, 2007), further supported the safety of this dose range.

The dose regimen was chosen because the loading dose (three doses in the first week) had resulted in creatinine increases in prior human studies (unpublished, L. van Meer et al.) and dedicated experiments in monkeys had shown that changes in renal markers occurred only with the loading-dose regimen. The treatment duration of 12 weeks (13 doses), which was expected to be safe and result in sufficient steady-state tissue concentrations, was selected on the basis of animal studies.

Clinical Measurements. Safety assessments, performed throughout the study period, included vital signs, electrocardiograms, physical examinations, and clinical laboratory tests [including clinical chemistry, hematology, coagulation, cytokines, complement tests, and urinalysis (including B2M and protein)] as well as registration of adverse events. Adverse events were defined as any new medical occurrence or worsening of a pre-existing condition after administration of the study drug or placebo. Predefined stopping rules regarding renal parameters were defined as changes in serum creatinine from a baseline of more than $0.3 \mathrm{mg} / \mathrm{dl}$ or more than $40 \%$ in 2 consecutive weeks, or proteinuria of more than $0.5 \mathrm{~g} / 24$ hours occurring in 2 consecutive weeks.

Renal Damage Markers. The biomarkers KIM1, cystatin C, epidermal growth factor (EGF), neutrophil gelatinase-associated lipocalin (NGAL)/LCN2, osteopontin, uromodulin, $\alpha$-glutathione $S$-transferase (aGST), and NAG were chosen on the basis of their performance in detecting injury to the proximal tubule, where SGLT2 is located (van Meer et al., 2014). Analysis of renal damage markers aGST and NAG was performed batchwise upon study completion by quantative enzyme immunoassays [Argutus Medical (Dublin, Ireland) NEPHKITO immunoassay for aGST, Diazyme (Poway, CA) 70010 Rev. F, colorimetric endpoint assay for NAG], and KIM-1, cystatin C, EGF, NGAL/LCN2, osteopontin, and uromodulin by enzyme-linked immunosorbent assay (R\&D Systems, Minneapolis, $\mathrm{MN})$.

Renal Perfusion and Glomerular Filtration. Renal clearance tests to assess renal plasma flow (RPF) and glomerular filtration rate (GFR) were performed using established and validated techniques (Rabelink et al., 1989; Toto, 1995; Honing et al., 2000). Sinistrin infusion allows calculation of GFR as it is not secreted or reabsorbed in any appreciable amount by the kidney. para-Aminohippurate (PAH) infusion allows calculation of RPF as it is completely secreted and not reabsorbed by the tubules. The intravenous infusion of sinistrin and $\mathrm{PAH}$ began 90 minutes after administration of ISIS 388626 or placebo. Infusion rates were calculated to obtain a steady-state concentration that was comparable between subjects and within the measurable range. $\mathrm{PAH}$ and sinistrin doses, corrected for lean body mass, serum creatinine, and age were administered via a continuous infusion of 120 minutes (infusion rates ranging from 400 to $750 \mathrm{mg} / \mathrm{h}$ and from 380 to $740 \mathrm{mg} / \mathrm{h}$ for sinistrin and $\mathrm{PAH}$, respectively), preceded by a 10-minute priming dose that was corrected for body surface area (ranging from 825 to $1200 \mathrm{mg}$ and from 840 to $1200 \mathrm{mg}$ for sinistrin 
and $\mathrm{PAH}$, respectively). Plasma samples for $\mathrm{PAH}$, sinistrin, and hematocrit measurement and urine samples for $\mathrm{PAH}$ and sinistrin measurement were collected at 30-minute intervals. During the infusion period, hydration was maintained by subjects drinking amounts of water matching urinary output, with a maximum of 4 liters, to ensure sufficient urine production. Serum and urinary sinistrin levels were analyzed according to the method described by Looye (1970). PAH levels were measured according to the method described by Waugh and Beall (1974).

No formal power calculation was performed; however, group size (12 treated subjects) was considered sufficient as expected size of changes in effects in GFR (in the present case) was around 17\%, and as previously shown, differences of $10 \%$ GFR can be detected with a group size of nine healthy volunteers (Honing et al., 2000).

Pharmacokinetics. ISIS 388626 plasma levels were measured frequently by a validated hybridization enzyme-linked immunosorbent assay (PDD Laboratories, Richmond, VA) for a 24-hour profile after the first and 13th ISIS 388626 doses, and predoses on weeks 3,8 , 10 during treatment and on five weekly follow-up visits. In addition, ISIS 388626 urine levels were measured using a validated capillary gel electrophoresis method (PPD Laboratories), in 24-hour collections after the first and 13th doses (up to 24 and 48 hours postdose).

Data Analysis and Statistical Methods. Safety and tolerability evaluations were made on the basis of descriptive statistics. ISIS388626 plasma concentrations were subjected to noncompartmental pharmacokinetic evaluation to determine the maximum observed plasma concentration $\left(C_{\max }\right)$, the time to maximum plasma concentration $\left(T_{\max }\right)$, the area under the plasma concentration-time curve from dosing to 24 hours after dosing $\left(\mathrm{AUC}_{0-24}\right.$ hours $)$ using WinNonLin (version 5.3; Pharsight Corporation, St. Louis, MO).

Pharmacodynamic evaluation was made on the basis of descriptive statistics as well as statistical analysis using analysis of covariance (ANCOVA) with baseline as a covariate. Endpoints were urinary glucose excretion and fractional glucose excretion [defined as urinary glucose excretion/filtered glucose load (GFR $\times$ fasted plasma glucose $\times 100$ ], and plasma glucose, insulin, and C-peptide concentrations. The selected sample sizes allowed explorative analysis of safety, pharmacokinetics, and pharmacodynamics of ISIS 388626 but were not supported by any statistical rationale.

\section{Results}

\section{Subjects}

Sixteen subjects participated in the first $50-\mathrm{mg} /$ placebo cohort. Another sixteen subjects were enrolled in the second $50-\mathrm{mg} /$ placebo cohort, which included renal clearance tests. Twenty-one subjects participated in the $100-\mathrm{mg} /$ placebo cohort, of which 15 subjects completed the study. Another
16 subjects participated in the $200-\mathrm{mg} /$ placebo cohort, of which 14 subjects completed the study. Subject demographics are presented in Table 1.

\section{Safety}

Adverse events (AEs) occurred in all subjects who received multiple doses of ISIS 388626 and in $85 \%$ of subjects who received placebo (Table 2). All AEs reported were classified as mild intensity and transient. The most common $\mathrm{AE}$ was nasopharingitis. Other AEs commonly reported in all groups were headache, fatigue, a range of gastrointestinal complaints (such as diarrhea, nausea, or abdominal discomfort), and musculoskeletal complaints (such as myalgia and back pain). These AEs occurred in the active treatment groups and placebo groups with a similar incidence; thus, it was considered improbable that they were ISIS 388626-related. Injection site reactions (ISRs) occurred at frequencies of $42 \%$ in the 50 -mg cohort, $19 \%$ in the $100-\mathrm{mg}$ cohort, and $8 \%$ in the $200-\mathrm{mg}$ cohort. ISRs consisted of mild erythema at the site of the subcutaneous injection without itch. In three subjects hyperpigmentation was reported after the initial erythema had resolved. In two subjects reappearance of erythema occurred after initial resolution. The ISRs were not progressive, not accompanied by local lymphadenopathy, and no study discontinuations occurred owing to ISRs. All ISRs resolved completely and spontaneously, ranging from within 12 hours to 50 days.

ISIS 388626 treatment did not result in any clinically relevant changes in vital signs (blood pressure, ECG-derived parameters, body temperature) or parameters of hematology and coagulation. No increases in circulating cytokines (interferon- $\alpha$, interleukin- 6 , monocyte chemoattractant protein1 , and macrophage inflammatory protein- $1 \alpha$ ) were observed at any dose level. Analysis of complement factors C5a and $\mathrm{Bb}$ revealed no changes, with the exception of an increase in factor $\mathrm{Bb}$ in the 200-mg dose group, maximally at 24 hours after the 13th dose (from $0.74 \pm 0.336 \mu \mathrm{g} / \mathrm{ml}$ at baseline to $0.93 \pm 0.394 \mu \mathrm{g} / \mathrm{ml}$ at 24 hours after dose of week 13).

Treatment with 50, 100, and $200 \mathrm{mg}$ of ISIS $388626 \mathrm{did}$ not result in clinically significant changes in such chemistry parameters as hepatic parameters and glucose levels, with the exception that in one participant after seven doses of $50 \mathrm{mg}$ of ISIS 388626 a transient increase in liver biochemistry parameters was observed (maximal change from baseline AST 3.6-fold ULN, ALT 4-fold ULN, confirmed by

TABLE 1

Summary of subject demographics

\begin{tabular}{lccccc}
\hline & Number & Age (Std) & BMI (Std) & Number female & $\begin{array}{c}\text { Early termination } \\
\text { of subjects }\end{array}$ \\
\hline & & $y r$ & $k g / \mathrm{m}^{2}$ & & \\
50 mg of ISIS 388626 & 12 & $33.9 \pm 14.23$ & $23.5 \pm 3.13$ & 0 & $2^{a}$ \\
50 mg with RCT & 12 & $35.2 \pm 14.68$ & $23.1 \pm 2.62$ & 1 & None \\
100 mg of ISIS 388626 & 16 & $37.5 \pm 16.18$ & $23.7 \pm 3.49$ & 0 & $5^{b}$ \\
200 mg of ISIS 388626 & 12 & $27.2 \pm 12.27$ & $23.7 \pm 2.82$ & 1 & $2^{c}$ \\
Pooled placebo & 13 & $36.1 \pm 16.18$ & $23.8 \pm 2.87$ & 1 & $1^{d}$ \\
Placebo with RCT & 4 & $31.3 \pm 8.06$ & $24.1 \pm 2.32$ & 0 & None \\
\hline
\end{tabular}

RCT, renal clearance tests.

${ }^{a}$ In one subject dosing was stopped after five doses owing to increases in serum creatinine and one subject was stopped after seven doses owing to increased liver biochemistry parameters.

${ }^{b}$ Stopped owing to personal reasons (after doses 1, 2, 4, 5, and 12). Subjects were replaced.

${ }^{c}$ Stopped (after seven and 10 doses) owing to increases in renal parameters.

${ }^{d}$ Stopped after one dose owing to personal reasons. Subject was replaced. 
TABLE 2

Frequency of adverse events reported in more than one subject

\begin{tabular}{|c|c|c|c|c|}
\hline & $50 \mathrm{mg}(n=12)$ & $100 \mathrm{mg}(n=16)$ & $200 \mathrm{mg}(n=12)$ & Placebo $(n=13)$ \\
\hline Nasopharyngitis & $42 \%(n=5)$ & $38 \%(n=6)$ & $67 \%(n=8)$ & $46 \%(n=6)$ \\
\hline Headache & $33 \%(n=4)$ & $56 \%(n=9)$ & $33 \%(n=4)$ & $38 \%(n=5)$ \\
\hline Mild gastrointestinal complaints & $58 \%(n=7)$ & $31 \%(n=5)$ & $42 \%(n=5)$ & $38 \%(n=5)$ \\
\hline Mild musculoskeletal complaints & $17 \%(n=2)$ & $25 \%(n=4)$ & $33 \%(n=4)$ & $38 \%(n=5)$ \\
\hline Fatigue & $33 \%(n=4)$ & $6 \%(n=1)$ & $8 \%(n=1)$ & $23 \%(n=3)$ \\
\hline ISRs & $42 \%(n=5)$ & $19 \%(n=3)$ & $8 \%(n=1)$ & $0 \%(n=0)$ \\
\hline
\end{tabular}

repeated measurement). This laboratory finding was suspected to be a viral infection as it coincided with mild joint pain and tonsillitis. Study-drug administration was discontinued and hepatic chemistry normalized within 2 weeks.

\section{Renal Safety}

Dose of $50 \mathrm{mg}$. Weekly dosing with $50 \mathrm{mg}$ of ISIS 388626 resulted in a rapid and sustained increase in serum creatinine concentrations, peaking at the end of dosing (Fig. 1A) with an average increase over baseline of $0.17 \pm 0.08 \mathrm{mg} / \mathrm{dl}$ $(+20 \%)$. The observed increase in serum creatinine was variable between subjects, ranging from 0.10 to $0.33 \mathrm{mg} / \mathrm{dl}$ after 13 doses, but the increase was observed in all ISIS 388626-treated subjects and in none of the placebo-treated subjects. Study drug administration was discontinued for one subject in whom one of the predefined stopping criteria was met: $41 \%$ increase in serum creatinine after five ISIS 388626 doses. Upon cessation of ISIS 388626 administration, serum creatinine levels returned to baseline in all subjects within 5 weeks. The observed changes in serum creatinine were not accompanied by rises in BUN or any clinically meaningful changes in serum electrolytes, albumin, aldosterone, or plasma renin activity (data not shown).

Urine flow and urinalysis parameters did not change significantly in the subjects with increased serum creatinine levels. ISIS 388626 treatment did not result in changes in renal damage markers NAG and aGST (included as Supplemental Fig. 1). Creatinine increases did coincide with increase in urinary B2M (Fig. 1C) with a maximal average change from baseline of $1250 \pm 1361 \mu \mathrm{g} / 24$ hours (15-fold increase) at week 12. Although the interindividual variability in urinary B2M was substantial, in nine out of 12 ISIS 388626-treated subjects an increase was observed, returning to baseline levels within 5 weeks after treatment cessation. Average excretion of urinary protein was larger in the ISIS 388626-treated group, but variability was substantial in both treatment groups (Fig. 1D).

To explore the nature of the observed renal findings in more detail, an additional cohort was treated with the same dose regimen, i.e., weekly administration of $50 \mathrm{mg}$ of ISIS 388626 or placebo. Renal clearance tests (PAH and sinistrin) were performed to assess kidney function. Comparable ISIS 388626-induced effects were observed as in the first study
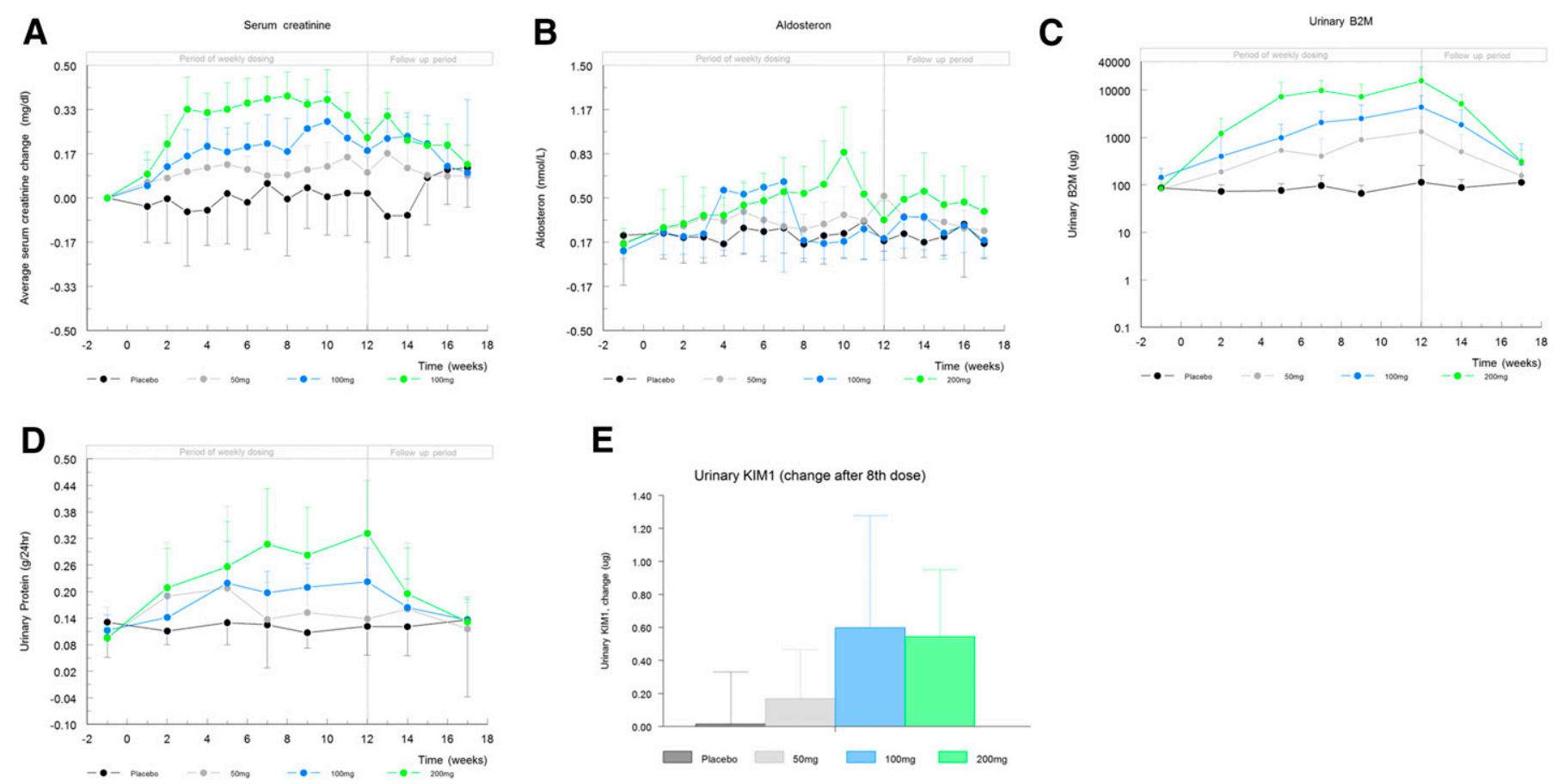

Fig. 1. Dose-dependent effects of ISIS 388626 on renal markers and recovery during the follow-up period. Average values with S.D. error bars. Logarithmic $Y$-axis used for B2M. Serum creatinine increases at all dose levels over time, expressed as change from baseline (A). Increases in absolute serum aldosterone for dose levels of 100 and $200 \mathrm{mg}$ (B). Increases in urinary B2M (C) and urinary protein (D) over time at all dose levels. Increases in urinary KIM1, expressed as change after the eighth dose compared with baseline (E). 

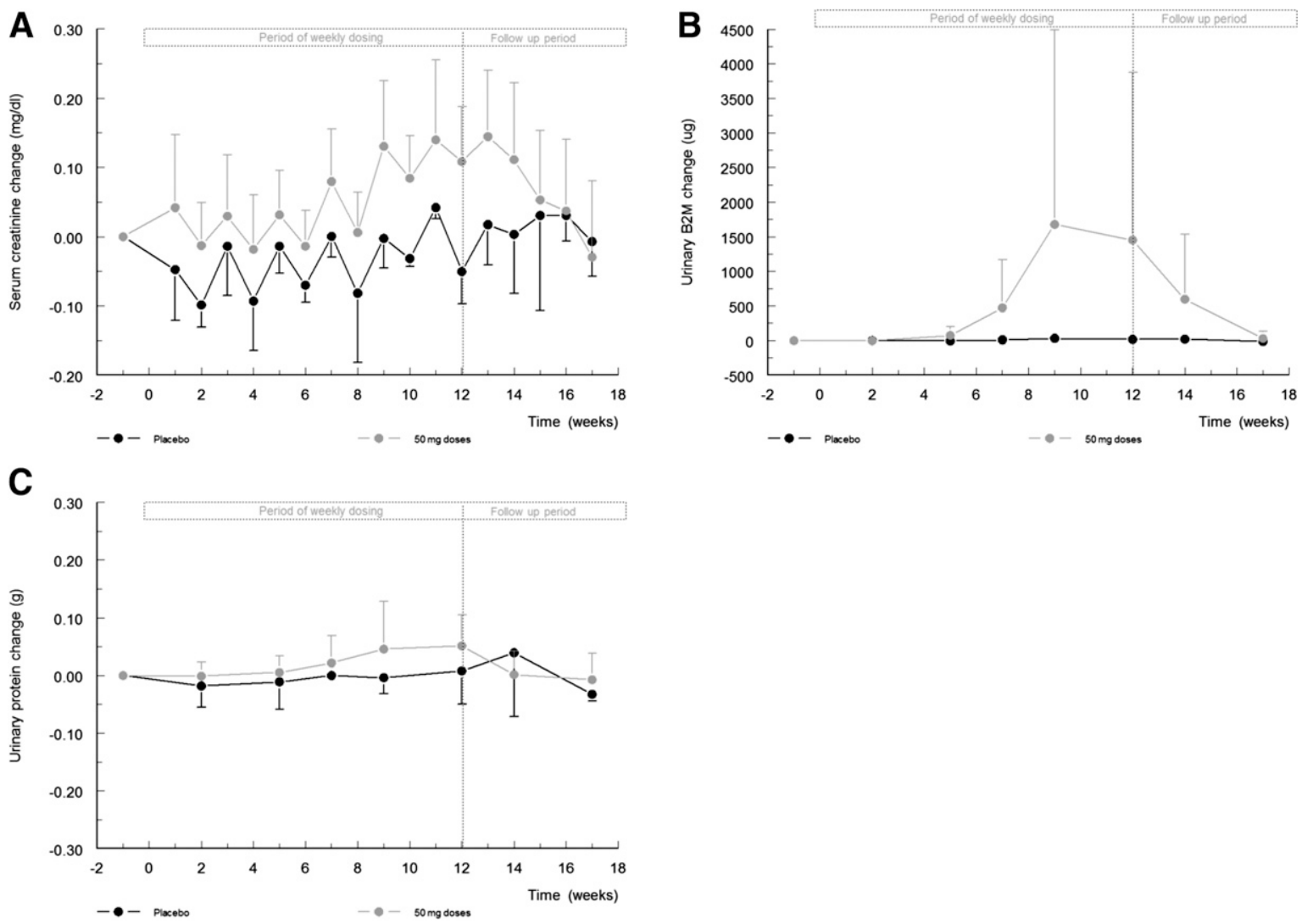

Fig. 2. Repeat study with $50 \mathrm{mg}$. Average values with S.D. error bars. Serum creatinine increases over time, expressed as change from baseline (A). Increases in urinary B2M (B) and urinary protein $(\mathrm{C})$.

part, with transient increases in serum creatinine and urinary $\mathrm{B} 2 \mathrm{M}$ and mildly elevated urinary protein levels (Fig. 2, A-C). $\mathrm{RPF}$ and GFR were in the expected range, as was the filtration fraction $(\mathrm{GFR} / \mathrm{RPF} \times 100)$ of approximately $20 \%$. Neither RPF nor GFR changed during the entire 13-week ISIS 388626 treatment period (Fig. 3).

Doses of 100 and $200 \mathrm{mg}$. Increasing exposure to ISIS 388626 led to further and dose-dependent changes in serum creatinine (Fig. 1A). Baseline serum creatinine levels were comparable between all treatment groups. The largest average increase of $0.38 \pm 0.089 \mathrm{mg} / \mathrm{dl}$ ( $44 \%$ increase) was reached after eight doses of $200 \mathrm{mg}$ of ISIS 388626. The effect of ISIS 388626 on serum creatinine was highly variable between subjects, ranging individually from no increase at all to a $0.49 \mathrm{mg} / \mathrm{dl}$ increase over baseline levels. Despite continued dosing, creatinine levels started to decline after ten doses, returning to baseline during the follow-up period. However, at the 200-mg dose average creatinine levels remained above baseline levels at week 17 . Three subjects met the protocolspecified stopping rule for renal function tests and were discontinued. The elevation in serum creatinine did not coincide with obvious changes in urea levels. At the highest
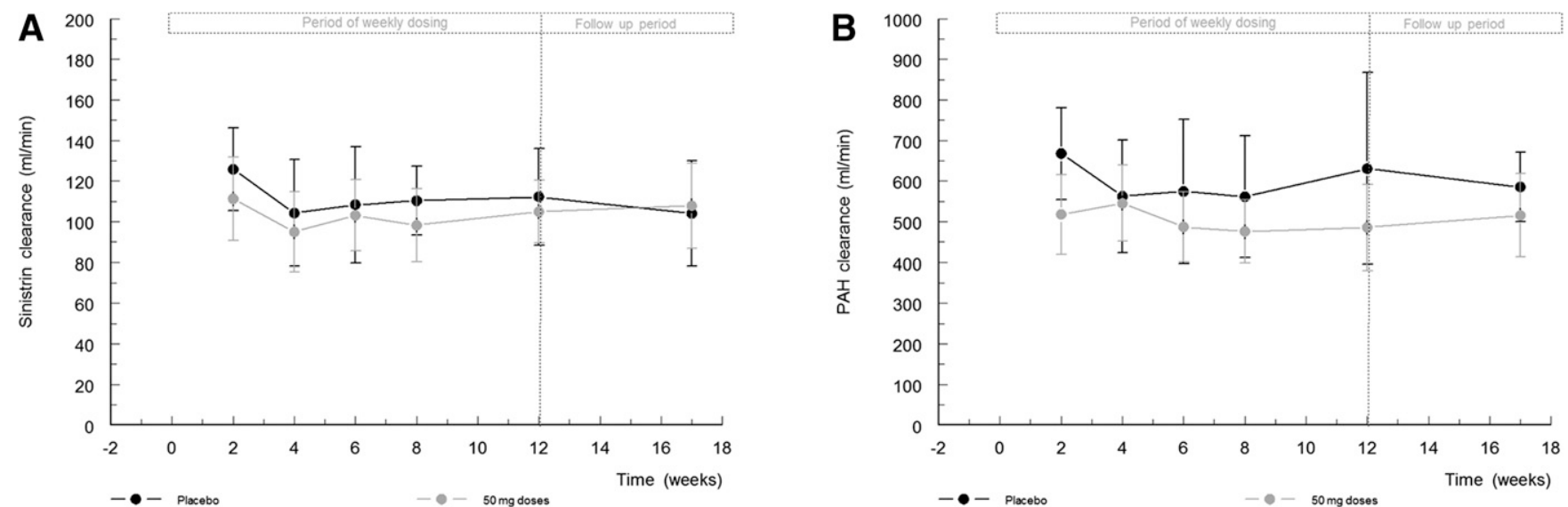

Fig. 3. Renal clearance tests. Average values with S.D. error bars. No changes observed in GFR, calculated by sinistrin clearance (A), and RPF, calculated by PAH clearance (B). 
dose level tested ISIS 388626 induced a short transient increase in aldosterone levels, although the inter- and intrasubject variability was significant (Fig. $1 \mathrm{~B}$ ).

ISIS 388626 treatment did not result in significant changes in urine flow and urinalysis parameters (data not shown) but did increase the levels of urinary renal damage markers (B2M, total protein, KIM1, aGST, NAG). ISIS 388626 treatment dose dependently increased urinary B2M (Fig. 1C). In all treatment groups B2M levels increased gradually with repeated ISIS 388626 administration, to decline after treatment stop during the 5-week follow-up period. Urinary protein also showed a dose-dependent increase, although the inter- and intrasubject variability was large (Fig. 1D). ISIS 388626 treatment, at all dose levels tested, resulted in an increase in urinary KIM1, assessed after the eighth administration of the compound (Fig. 1E). Urinary levels of aGST and NAG also increased upon ISIS 388626 treatment (included as Supplemental Fig. 1).

\section{Pharmacodynamics}

ISIS 388626 dose dependently increased 24-hour urinary glucose excretion (Fig. 4A, to $294.6 \pm 489.65$, $575.6 \pm 789.5$, and $1413.1 \pm 1804.6 \mathrm{mg} /$ day glucose excretion at end of treatment in the 50-, 100-, and 200-mg cohorts, versus $172.3 \pm$ $387.0 \mathrm{mg} / \mathrm{day}$ in the placebo group). During the 5 -week follow-up period, values returned to baseline, although not completely for the 200-mg cohort. Average values of fractional glucose excretion, calculated from 24-hour urine glucose excretion and serum glucose, showed a very similar pattern (included as Supplemental Fig. 2).
The OGTT resulted in an expected strong increase in serum glucose, insulin, and C-peptide levels, followed by a rapid decline toward normal values (data not shown). ISIS 388626 treatment did not affect the OGTT-induced increase in serum glucose. ISIS 388626 treatment resulted in a dose-dependent enhancement of insulin and C-peptide release (Fig. 4, B and C). Urinary glucose excretion during the first 4 hours of the OGTT was increased in ISIS 388626-treated groups compared with placebo (Fig. 4D, 256.6 \pm 445.97, 347.3 \pm 411.4, and $745.6 \pm$ $1122.4 \mathrm{mg} / 4$ hours for the 50-, 100-, and 200-mg cohorts, versus $133.9 \pm 327.5 \mathrm{mg} / 4$ hours in the placebo groups). Increases were statistically different from placebo.

\section{Pharmacokinetics}

ISIS 388626 rapidly entered the circulation upon subcutaneous injection, with maximum plasma concentrations $\left(C_{\max }\right)$ occurring within the first 2 hours after dosing and rapidly declining concentrations thereafter (Fig. 5, A-C and Table 3). $C_{\max }$ and area under the plasma concentration time curve ( $\mathrm{AUC}_{0-24}$ hours, total exposure) showed no accumulation of ISIS 388626 upon repeated administration at dose levels of 50 and $100 \mathrm{mg}$. Some accumulation might have been present at $200 \mathrm{mg}$ as $C_{\max }$ and AUC increased slightly from the first to the 13th dose (Fig. 5C, Table 3).

The total amount of ISIS 388626 in urine (0-24 hours) ranged from 3.7 to $18.4 \mathrm{mg}$ over the dose range tested and increased in a dose-proportional manner (Fig. 5D). Urinary excretion was approximately 2 -fold higher after the 13 th dose compared with the first dose.

\section{A}

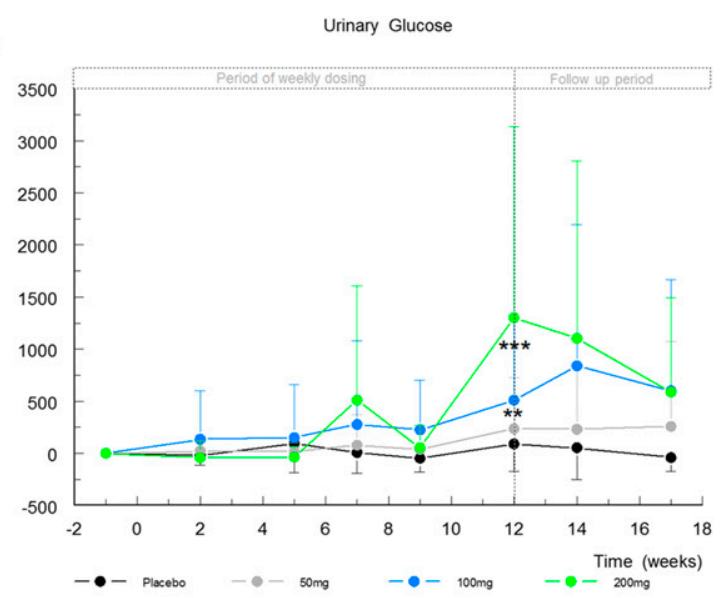

B

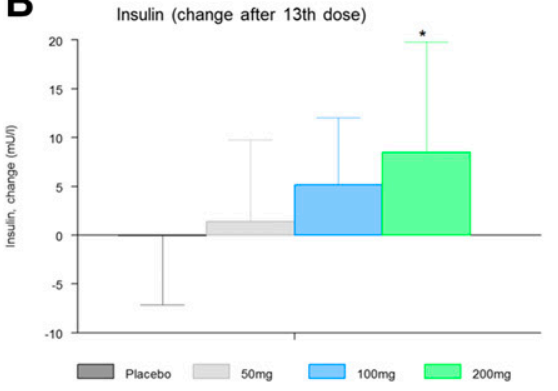

C

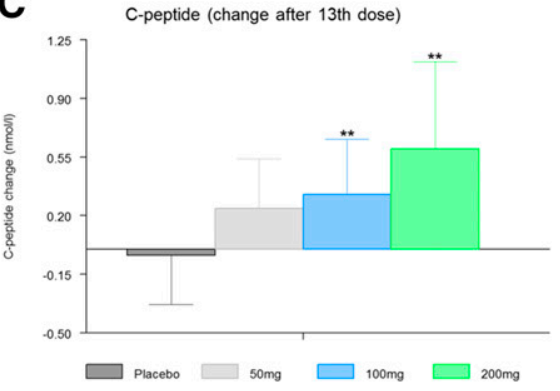

D

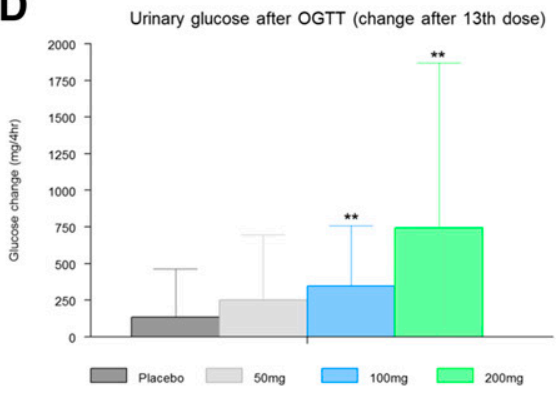

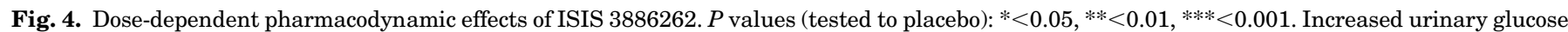

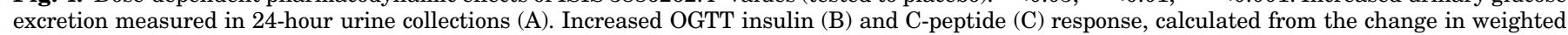

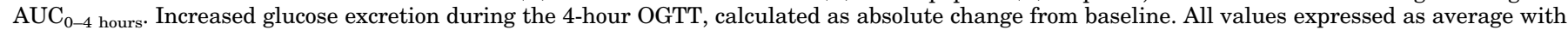
S.D. error bars. 
A mean ISIS 388626 concentrations in plasma

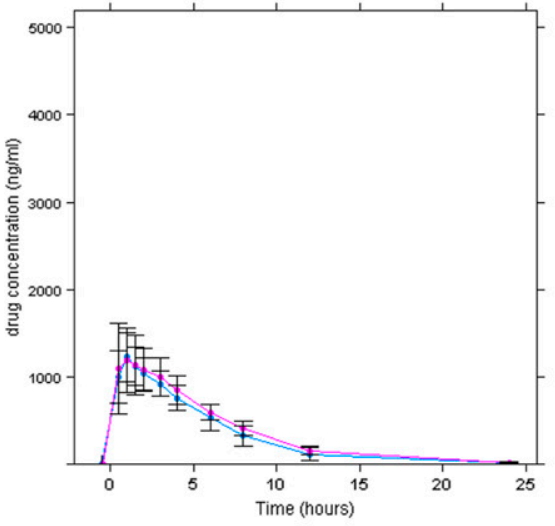

Urinary ISIS 388626

D

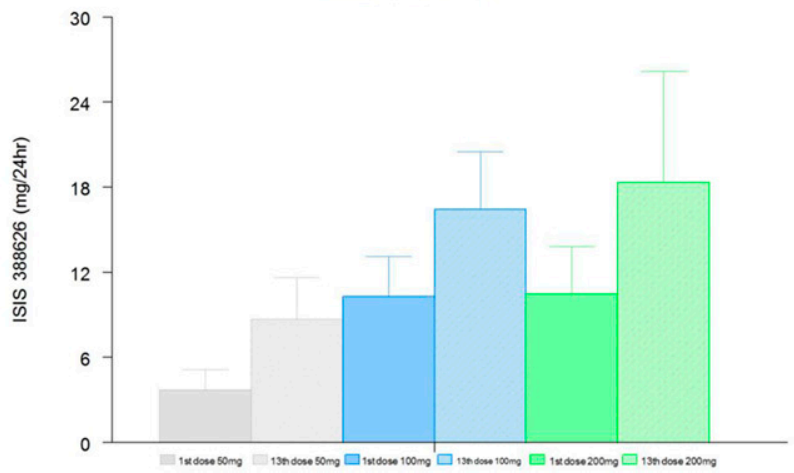

B mean ISIS 388626 concentrations in plasma Dose $=100 \mathrm{mg}$

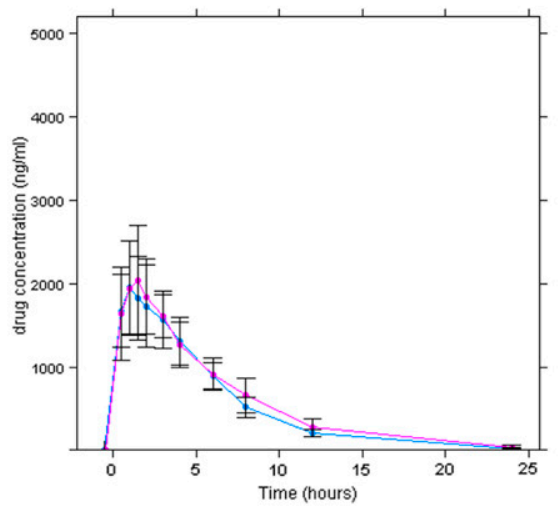

C mean ISIS 388626 concentrations in plasma

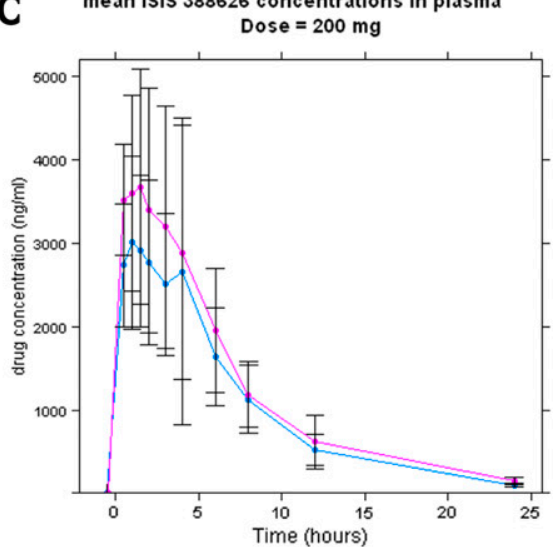

Fig. 5. Pharmacokinetic properties of ISIS 388626. The blue line represents the values measured after the first dose, the purple line after the 13th dose. Average values with S.D. error bars. Rapid absorption upon injection $\left(C_{\max }\right.$ within 2 hours $)$ and rapidly declining concentrations thereafter at all dose levels (A-C). No accumulation at dose levels of $50 \mathrm{mg}$ and $100 \mathrm{mg}$, possible accumulation for $200 \mathrm{mg}$, with an increased AUC after the 13th dose. (D) Twenty-four excretion of ISIS 388626 in urine increased proportionally to dose, with an increased excretion after the 13th dose compared with the first dose.

\section{Discussion}

The antisense oligonucleotide ISIS 388626, targeting the renal SGLT2 receptor, did not induce pharmacological effects (i.e., induction of glucosuria) when dose levels up to $50 \mathrm{mg}$ were applied, with weekly dosing for 12 weeks. This was not unexpected since in preclinical studies across multiple species, the pharmacologically active doses were doses exceeding $1 \mathrm{mg} / \mathrm{kg}$ per week, which translates into $70 \mathrm{mg} / \mathrm{kg}$ per week assuming an average weight of $70 \mathrm{~kg}$ (Wancewicz, 2008; Bhanot, 2009; Zanardi et al., 2012). Repeated ISIS 388626 administration with $50 \mathrm{mg}$ did induce transient and mild rises in serum creatinine. To explore whether the observed transient increases in renal markers coincided with functional changes, a new cohort of volunteers was exposed to the same ISIS 388626 dose level and regimen, and PAH and sinistrin clearances were assessed to estimate RPF and GFR. The effects on serum creatinine and urinary markers were reproduced in this second cohort, but we found no indications that the changes can be explained by ISIS 388626-induced changes in GFR and RPF. This allowed dose escalation to possibly elicit pharmacological effects.

ISIS 388626 dose dependently increased urinary glucose excretion. After 13 weekly doses urinary glucose increased, which ceased after therapy cessation. On average, the maximal level of urinary glucose excretion was $1.4 \mathrm{~g}$ per day, observed in the 200-mg ISIS 388626 treatment group after 13 weekly doses, compared with $0.2 \mathrm{~g}$ in the placebo group (7-fold increase). The observations suggest an ISIS 388626induced increase in glucosuria, but the effect was rather small, since the average amount of glucose that is filtered daily is approximately $144 \mathrm{~g}$ [800 mmol/day (Berne et al., 1998)]. Furthermore, compared with treatment with smallmolecule SGLT2 inhibitors, the observed level of urinary glucose excretion was minimal, as these compounds induce

TABLE 3

Plasma pharmacokinetics after first and 13th doses

\begin{tabular}{lccccrc}
\hline & \multicolumn{2}{c}{$50 \mathrm{mg}$} & \multicolumn{2}{c}{$100 \mathrm{mg}$} & $200 \mathrm{mg}$ \\
\hline After dose no. & 1 & 13 & 1 & 13 & 1 & 13 \\
$N$ & 12 & 10 & 16 & 12 & 9 \\
$\mathrm{AUC}_{0-24 \text { hours }}(\mu \mathrm{g} \times$ hour $/ \mathrm{ml})$ & $7627 \pm 1202$ & $8626 \pm 1037$ & $12,650 \pm 2085$ & $13,970 \pm 2041$ & $24,250 \pm 8796$ & $28,320 \pm 9875$ \\
$C_{\max }(\mu \mathrm{g} / \mathrm{ml})$ & $1240 \pm 274.4$ & $1275 \pm 470.6$ & $2048 \pm 487.5$ & $2185 \pm 558.2$ & $3333 \pm 1602$ & $4155 \pm 1266$ \\
$T_{\max }($ hours $)$ & $1.17 \pm 0.324$ & $1.40 \pm 0.566$ & $1.38 \pm 0.97$ & $1.50 \pm 0.88$ & $1.63 \pm 1.11$ & $1.56 \pm 1.37$ \\
\hline
\end{tabular}


urinary glucose excretion in the range of 50-80 g per day (Kasichayanula et al., 2014). In spite of the relatively small effect observed, the findings do suggest that ISIS 388626 doses of $200 \mathrm{mg}$ and beyond exert intended pharmacodynamic activity. Animal data demonstrated that a certain threshold level of SGLT2 mRNA exists, maintaining tubular glucose reabsorption and limiting glucosuria, and that once this threshold is exceeded, glucose reabsorption strongly declines, resulting in sudden increases in glucosuria: An ISIS 388626mediated SGLT2 mRNA reduction of nearly $70 \%$ did not induce a significant increase in glucosuria, whereas an SGLT2 mRNA reduction of $85 \%$ resulted in a 100 -fold increase in glucosuria (data on file, monkey treated with $2 \mathrm{mg} / \mathrm{kg}$ per week versus $24 \mathrm{mg} / \mathrm{kg}$ per week ISIS 388626). This is supported by the observation that a phenotype of hereditary SGLT2 mutations with 50-60\% loss of SGLT2 does not display significant glucosuria (Santer et al., 2003). Compensation by an increase in SGLT1-mediated transport would probably play a role in the maintenance of renal glucose reabsorption, as was demonstrated in animals after genetic and pharmacological SGLT2 inhibition (Rieg et al., 2014).

In line with the mild glucosuria observed in 24-hour urine collections, urinary glucose excretion after the OGTT also increased in the ISIS 388626-treated groups. Furthermore, ISIS 388626 treatment resulted in elevated levels of circulating insulin and C-peptide during the first hours after the glucose load. These increases seem counter-intuitive, as increased urinary glucose loss is expected to result in a lowering of insulin levels needed to compensate for the glucose load. Probably, changes in other mechanisms involved in glucose homeostasis occur simultaneously. Treatment with SGLT2 inhibitors dapagliflozin and empagliflozin resulted in similar paradoxical findings of altered glucose homeostasis and increased C-peptide/insulin levels, probably compensating for an increased endogenous glucose production (Cefalu, 2014; Ferrannini et al., 2014; Merovci et al., 2014; Scheen and Paquot, 2014).

Although the observed glucosuria after ISIS 388626 treatment may result from antisense-mediated inhibition of SGLT2 in humans, it may also relate to ISIS 388626-induced tubular dysfunction. At the highest dose level, ISIS 388626 treatment resulted in increases in serum creatinine levels of 40-50\% over baseline, with concomitant induction of other markers indicative of renal damage or dysfunction. The correlation between induction of renal damage markers and glucosuria was explored (Table 4), and demonstrated a correlation between the renal markers serum creatinine, urinary $\mathrm{B} 2 \mathrm{M}$, and urinary protein (with coefficients of 0.46 , 0.47 , and 0.66 , Table 4 ), but no correlation with urinary glucose excretion (with coefficients of $0.086,0.43$, and 0.23 ,

\section{TABLE 4}

Correlation coefficients of the exploratory analysis of correlations between serum creatinine (s.creat), urinary B2M (u.B2M), urinary protein (u.prot), and urinary glucose (u.gluc), calculated with change from baseline values (CFB)

\begin{tabular}{lccc}
\hline & CFB s.creat & CFB u.B2M & CFB u.prot \\
\hline CFB s.creat & $\mathrm{X}$ & $0.46^{* *}$ & $0.47^{* *}$ \\
CFB u.B2M & $0.46^{* *}$ & $\mathrm{X}$ & $0.66^{* *}$ \\
CFB u.prot & $0.47^{* *}$ & $0.66^{* *}$ & $\mathrm{X}$ \\
CFB u.gluc & 0.086 & 0.43 & 0.23 \\
\hline
\end{tabular}

**Positive correlation considered significant.
Table 4). The absence of a correlation between renal side effects and intended pharmacodynamic effect suggests that the observed mild glucosuria probably results from antisensemediated inhibition of SGLT2, and not from tubular dysfunction. Moreover, it makes it improbable that a general membrane dysfunction occurs as a result of knockdown of the SGLT2 receptor. This is further supported by the absence of signs of membrane dysfunction with other oligonucleotides are directed to transmembrane receptors (Limmroth et al., 2014; van Dongen et al., 2015). ISIS 388626 is, however, the first antisense that oligonucleotide targeted to a renal receptor, and it remains uncertain if knockdown interferes with membrane function. On the other hand, subjects with homozygous SGLT2 mutations are largely asymptomatic and have no signs of renal tubular dysfunction, hypovolemia, or electrolyte imbalance (Francis et al., 2004).

We hypothesize that the changes in serum creatinine and urinary renal markers induced by ISIS 388626 treatment most probably reflect transient tubular dysfunction. Although urinary B2M and protein may also increase in the case of glomerular injury owing to increased filtration (Hoffmann et al., 2010), the reversible nature of the changes in our study suggest interference with tubular reabsorption. This is supported by the observed elevated levels of KIM1, aGST, and NAG, all markers known in animal models to increase in response to different tubulo-toxic agents (Feinfeld et al., 1986; Hoffmann et al., 2010; Vaidya et al., 2010; Ouchi et al., 2012; Swain et al., 2012 ). Accumulation of antisense oligonucleotides occurs in proximal tubular cells as basophilic granules (Monteith et al., 1999; Henry et al., 2012), and this was indeed seen also in animal studies with ISIS 388626 (Zanardi et al., 2012). In primates, tubular accumulation of oligonucleotides is usually not associated with renal toxicity and tubular functional changes, unless extremely high doses are used (Monteith et al., 1999; Henry et al., 2012; Zanardi et al., 2012 ). In accordance, these compounds are not associated with adverse renal effects in humans during subsequent clinical investigations (Kastelein et al., 2006; van Dongen et al., 2015). However, it is of note that, compared with other 18- to 20-mer second-generation oligonucleotides, the 12-mer chemistry of ISIS 388626 enables more selective distribution to the kidney (Wancewicz, 2008), the result of lower plasma protein binding, higher free fraction, and increased renal filtration. It is uncertain if this increased selectivity contributes to the observed adverse renal effects, but it should be taken in account that this is a theoretical possibility. No other comparable 12-mer oligonucleotides have been investigated in humans to date. Despite the observed increases in renal markers, no functional loss occurred at weekly doses of $50 \mathrm{mg}$, as demonstrated in the dedicated cohort, with renal clearance tests supporting the hypothesis that the observed changes reflect adaptation and possibly regeneration of tubular cells. Also, no trend was detected in regression analysis exploring the relation between values of GFR/RPF and renal damage markers (data not shown). Examples of other drugs affecting tubular creatinine secretion without decreasing glomerular filtration rate include cimetidine and pyrimethamine (Andreev et al., 1999). Finally, the observation that the effect of ISIS 366828 on serum creatinine diminishes after ten doses, despite continued dosing, supports the likelihood that antisense treatment is associated with a process of tubular adaptation. 
Treatment with small-molecule SGLT2 inhibitors also increases serum creatinine (http://www.fda.gov/downloads/ AdvisoryCommittee/UCM262994.pdf; http://www.fda.gov/ downloads/AdvisoryCommittees/CommitteesMeetingMaterials/ Drugs/EndocrinologicandMetabolicDrugsAdvisoryCommittee/ UCM334550.pdf). However, these effects were smaller and considered secondary to volume depletion owing to osmotic diuresis. These mild effects on serum creatinine coincided with a much larger pharmacological effect; therefore, this does not provide sufficient explanation for our findings. Renal side effects are commonly observed for clinically tested oligonucleotide compounds, such as PRO051 [developed for Duchenne Muscular Dystrophy, and associated with proteinuria (Goemans et al., 2011)], LY2181308, [developed for treatment of melanoma and the cause of a case of reversible kidney damage (Herrington et al., 2011)], and SPC5001 [developed for familial hypercholesterolemia, associated with increased renal markers and a case of acute tubular necrosis (Van Poelgeest et al., 2013)]. Although these observations suggest an unintended class effect of oligonucleotides, several other antisense compounds with chemical characteristics comparable to ISIS 388626 were free of renal side effects in humans, such as mipomersen (Santos et al., 2013), ISIS 325568 (van Dongen et al., 2015), and ISIS 2302 and ISIS 104838 (Kwoh, 2007). Recently, it has been shown in monkeys that chronic administration of a compound similar in class, drisapersen, results in C3 glomerulopathy most probably the result of an mechanism with an immune basis (Frazier et al., 2013). This appears to be consistent with the observation that antisense oligonucleotides may activate the alternative pathway of the complement system via transient inhibition of factor $\mathrm{H}$ (Henry et al., 1997).

\section{Conclusion}

Taken together, our data suggest that ISIS 388626 exerts its intended pharmacological effect in humans: Treatment of a period of 12 weeks at a dose level of weekly $200 \mathrm{mg}$ resulted in a small but significant elevation in urinary glucose. Concomitantly, changes in serum and urinary markers were indicative of transient renal dysfunction, most probably of tubular origin. Theoretically, the ISIS 388626-induced tubular dysfunction may have contributed to the glucosuria, but as the increases in renal markers do not correlate with the glucosuria, the latter is more probably an effect of oligonucleotide-induced SGLT2 inhibition. The pharmacodynamic effect of ISIS 388626 in type 2 diabetes mellitus patients, with elevated SGLT2 expression and a larger renal glucose load, may outweigh the effect observed in healthy volunteers. However, the efficacy is probably insufficient with the dose levels so far tested, considering the glucosuria observed with existing small-molecule SGLT2 inhibitors. Moreover, the mechanisms underlying the transient renal dysfunction warrant more detailed exploration. In the first place, because the target patient population are subjects with diabetes type 2 , who are known to be particularly prone to renal injury, chronic kidney disease may develop as a result. And secondly, as we know renal effects are also observed for other oligonucleotides, this might prohibit use of this promising drug class for chronic use in conditions that are not directly life threatening.

\section{Authorship Contributions}

Participated in research design: van Meer, van Dongen, Cohen, Burggraaf.

Conducted experiments: van Meer, van Dongen, Cohen, Burggraaf. Performed data analysis: van Meer, Goulouze, Klaassen.

Wrote or contributed to the writing of the manuscript: van Meer, Moerland, Cohen, Burggraaf.

\section{References}

Andreev E, Koopman M, and Arisz L (1999) A rise in plasma creatinine that is not a sign of renal failure: which drugs can be responsible? J Intern Med 246:247-252. Berne R, Levy M, Koeppen B, and Stanton B. Physiology. 4th, 699. 1998.

Bhanot S (2009) ISIS 388626, an SGLT2 antisense drug, causes robust and sustained glucosuria in multiple species and is safe and well-tolerated [abstract 328-OR] Diabetes Pro (American Diabetes Association) http://professional.diabetes.org/ abstract/isis-388626-sglt2-antisense-drug-causes-robust-and-sustained-glucosuriamultiple-species (acccessed 27 August 2016).

Cefalu WT (2014) Paradoxical insights into whole body metabolic adaptations following SGLT2 inhibition. J Clin Invest 124:485-487.

Feinfeld DA, Fuh VL, and Safirstein R (1986) Urinary glutathione-S-transferase in cisplatin nephrotoxicity in the rat. J Clin Chem Clin Biochem 24:529-532.

Ferrannini E, Muscelli E, Frascerra S, Baldi S, Mari A, Heise T, Broedl UC, and Woerle HJ (2014) Metabolic response to sodium-glucose cotransporter 2 inhibition in type 2 diabetic patients. $J$ Clin Invest 124:499-508.

Francis J, Zhang J, Farhi A, Carey H, and Geller DS (2004) A novel SGLT2 mutation in a patient with autosomal recessive renal glucosuria. Nephrol Dial Transplant 19:2893-2895.

Frazier KS, Sobry C, Derr V, Adams MJ, Besten CD, Kimpe SD, Francis I, Gales TL, Haworth R, Maguire SR, et al. (2013) Species-specific inflammatory responses as a primary component for the development of glomerular lesions in mice and monkeys following chronic administration of a second-generation antisense oligonucleotide. Toxicol Pathol 42:923-935.

Goemans NM, Tulinius M, van den Akker JT, Burm BE, Ekhart PF, Heuvelmans N, Holling T, Janson AA, Platenburg GJ, Sipkens JA, et al. (2011) Systemic administration of PRO051 in Duchenne's muscular dystrophy. $N$ Engl J Med 364: $1513-1522$

Henry SP, Bolte H, Auletta C, and Kornbrust DJ (1997) Evaluation of the toxicity of ISIS 2302, a phosphorothioate oligonucleotide, in a four-week study in cynomolgus monkeys. Toxicology 120:145-155.

Henry SP, Johnson M, Zanardi TA, Fey R, Auyeung D, Lappin PB, and Levin AA (2012) Renal uptake and tolerability of a 2'-O-methoxyethyl modified antisense oligonucleotide (ISIS 113715) in monkey. Toxicology 301:13-20.

Herrington WG, Talbot DC, Lahn MM, Brandt JT, Callies S, Nagle R, Winearls CG, and Roberts IS (2011) Association of long-term administration of the survivin mRNA-targeted antisense oligonucleotide LY2181308 with reversible kidney injury in a patient with metastatic melanoma. Am J Kidney Dis 57:300-303.

Hoffmann D, Fuchs TC, Henzler T, Matheis KA, Herget T, Dekant W, Hewitt P, and Mally A (2010) Evaluation of a urinary kidney biomarker panel in rat models of acute and subchronic nephrotoxicity. Toxicology 277:49-58.

Honing ML, Hijmering ML, Ballard DE, Yang YP, Padley RJ, Morrison PJ, and Rabelink TJ (2000) Selective ET(A) receptor antagonism with ABT-627 attenuates all renal effects of endothelin in humans. J Am Soc Nephrol 11: 1498-1504

Jahagirdar V and Barnett AH (2014) Empagliflozin for the treatment of type 2 diabetes. Expert Opin Pharmacother 15:2429-2441.

Kasichayanula S, Liu X, Lacreta F, Griffen SC, and Boulton DW (2014) Clinical pharmacokinetics and pharmacodynamics of dapagliflozin, a selective inhibitor of sodium-glucose co-transporter type 2. Clin Pharmacokinet 53:17-27

Kastelein JJ, Wedel MK, Baker BF, Su J, Bradley JD, Yu RZ, Chuang E, Graham MJ, and Crooke RM (2006) Potent reduction of apolipoprotein B and low-density lipoprotein cholesterol by short-term administration of an antisense inhibitor of apolipoprotein B. Circulation 114:1729-1735.

Kwoh TJ (2007) An overview of clinical safety experience of first- and secondgeneration antisense oligonucleotides, in Antisense Drug Technology: Principles, Strategies, and Application (Crooke ST ed), 365-399, CRC Press, Boca Raton, FL.

Levey AS, Bosch JP, Lewis JB, Greene T, Rogers N, and Roth D; Modification of Diet in Renal Disease Study Group (1999) A more accurate method to estimate glomerular filtration rate from serum creatinine: a new prediction equation. Ann Intern Med 130:461-470.

Limmroth V, Barkhof F, Desem N, Diamond MP, and Tachas G; ATL1102 Study Group (2014) CD49d antisense drug ATL1102 reduces disease activity in patients with relapsing-remitting MS. Neurology 83:1780-1788.

Looyé A (1970) Automated simultaneous determination of p-acetylaminohippurate and inulin in serum. Clin Chem 16:753-755.

Merovci A, Solis-Herrera C, Daniele G, Eldor R, Fiorentino TV, Tripathy D, Xiong J, Perez Z, Norton L, Abdul-Ghani MA, et al. (2014) Dapagliflozin improves muscle insulin sensitivity but enhances endogenous glucose production. $J$ Clin Invest 124: $509-514$.

Monteith DK, Horner MJ, Gillett NA, Butler M, Geary R, Burckin T, UshiroWatanabe T, and Levin AA (1999) Evaluation of the renal effects of an antisense phosphorothioate oligodeoxynucleotide in monkeys. Toxicol Pathol 27:307-317.

Ouchi M, Suzuki T, Hashimoto M, Motoyama M, Ohara M, Suzuki K, Igari Y, Watanabe K, Nakano H, and Oba K (2012) Urinary N-acetyl- $\beta$-D-glucosaminidase levels are positively correlated with 2 -hr plasma glucose levels during oral glucose tolerance testing in prediabetes. J Clin Lab Anal 26:473-480.

Plosker GL (2012) Dapagliflozin: a review of its use in type 2 diabetes mellitus. Drugs 72:2289-2312. 
Rabelink AJ, Koomans HA, Boer WH, Dorhout Mees EJ, and van Rijn HJ (1989) Indomethacin increases renal lithium reabsorption in man. Nephrol Dial Transplant 4:27-31.

Rieg T, Masuda T, Gerasimova M, Mayoux E, Platt K, Powell DR, Thomson SC, Koepsell H, and Vallon V (2014) Increase in SGLT1-mediated transport explains renal glucose reabsorption during genetic and pharmacological SGLT2 inhibition in euglycemia. Am J Physiol Renal Physiol 306:F188-F193.

Santer R, Kinner M, Lassen CL, Schneppenheim R, Eggert P, Bald M, Brodehl J, Daschner M, Ehrich JH, Kemper M, et al. (2003) Molecular analysis of the SGLT2 gene in patients with renal glucosuria. J Am Soc Nephrol 14:2873-2882.

Santos RD, Duell PB, East C, Guyton JR, Moriarty PM, Chin W, and Mittleman RS (2013) Long-term efficacy and safety of mipomersen in patients with familial hypercholesterolaemia: 2-year interim results of an open-label extension. Eur Heart $J$ 36:566-575.

Scheen AJ and Paquot N (2014) Metabolic effects of SGLT-2 inhibitors beyond increased glucosuria: A review of the clinical evidence. Diabetes Metab 40(6, Suppl 1): S4-S11.

Sha S, Devineni D, Ghosh A, Polidori D, Hompesch M, Arnolds S, Morrow L, Spitzer H, Demarest K, and Rothenberg P (2014) Pharmacodynamic effects of canagliflozin, a sodium glucose co-transporter 2 inhibitor, from a randomized study in patients with type 2 diabetes. PLoS One 9:e110069.

Swain A, Turton J, Scudamore C, Maguire D, Pereira I, Freitas S, Smyth R, Munday M, Stamp C, Gandhi M, et al. (2012) Nephrotoxicity of hexachloro-1:3-butadiene in the male Hanover Wistar rat; correlation of minimal histopathological changes with biomarkers of renal injury. J Appl Toxicol 32:417-428.

Toto RD (1995) Conventional measurement of renal function utilizing serum creatinine, creatinine clearance, inulin and para-aminohippuric acid clearance. Curr Opin Nephrol Hypertens 4:505-509, discussion 503-504

Vaidya VS, Ozer JS, Dieterle F, Collings FB, Ramirez V, Troth S, Muniappa N, Thudium D, Gerhold D, Holder DJ, et al. (2010) Kidney injury molecule-1 outperforms traditional biomarkers of kidney injury in preclinical biomarker qualification studies. Nat Biotechnol 28:478-485.

van Dongen MG, Geerts BF, Morgan ES, Brandt TA, de Kam ML, Romijn JA, Cohen AF, Bhanot S, and Burggraaf J (2015) First proof of pharmacology in humans of a novel glucagon receptor antisense drug. J Clin Pharmacol 55:298-306.

van Poelgeest EP, Swart RM, Betjes MGH, Moerland M, Weening JJ, Tessier Y, Hodges MR, Levin AA, and Burggraaf J (2013) Acute kidney injury during therapy with an antisense oligonucleotide directed against PCSK9. Am J Kidney Dis 62: 796-800.

van Meer L, Moerland M, Cohen AF, and Burggraaf J (2014) Urinary kidney biomarkers for early detection of nephrotoxicity in clinical drug development. $\mathrm{Br} J$ Clin Pharmacol 77:947-957.

Wancewicz EV (2008) Long term safety and efficacy of ISIS 388626, an optimized SGLT2 antisense inhibitor, in multiple diabetic and euglycemic species [abstract 334-OR]. Diabetes Pro (American Diabetes Association) http://professional. diabetes.org/abstract/long-term-safety-and-efficacy-isis-388626-optimized-sglt2 antisense-inhibitor-multiple (accessed 27 August 2016).

Waugh WH and Beall PT (1974) Simplified measurement of p-aminohippurate and other arylamines in plasma and urine. Kidney Int 5:429-436.

Zanardi TA, Han SC, Jeong EJ, Rime S, Yu RZ, Chakravarty K, and Henry SP (2012) Pharmacodynamics and subchronic toxicity in mice and monkeys of ISIS 388626, a second generation antisense oligonucleotide that targets the human sodium glucose cotransporter 2 (SGLT2). J Pharmacol Exp Ther 343:489-496

Address correspondence to: Dr. M. Moerland, Centre for Human Drug Research, Zernikedreef 8, 2333CL Leiden, The Netherlands. E-mail: mmoerland@chdr.nl 